\title{
INVESTIGATION OF BOUNDARY CONDITION EFFECTS ON THE STABILITY OF FGP BEAMS IN THERMAL ENVIRONMENT
}

\author{
Reza Nasirzadeh, Bashir Behjat, Mahsa Kharazi, Ata Khabazaghdam \\ Mechanical Engineering Faculty, Sahand University of Technology, Tabriz, Iran \\ e-mail: behjat@sut.ac.ir
}

\begin{abstract}
In this paper, stability and instability of Functionally Graded Piezoelectric (FGP) beams is investigated based on the Timoshenko beam theory. The material properties of the beam are considered to change gradually through thickness of the beam by a simple power law. By using the principle of minimum total potential energy, governing equations of the beam are derived. Stability behavior of the beam is predicted by solving the governing equations of the FGP beam. The results show that the homogeneity of boundary conditions plays a critical role in the stability of the FGP beam. While non-homogeneous boundary conditions lead to stable behavior of the FGP beam; homogeneous boundary conditions cause instability in the beam. By solving the eigenvalue equation of the FGP beam, the buckling load of the beam is obtained for the beams that have unstable behavior. Finally, the effects of various parameters on the buckling load of the unstable beam, such as power law index, temperature, applied voltage and aspect ratio are investigated, and the results are compared with the Euler-Bernoulli beam theory.
\end{abstract}

Keywords: FGP beam, stability, instability, buckling load, Timoshenko beam theory, non-homogeneous and homogeneous boundary conditions

\section{Introduction}

Piezoelectric materials have been commonly used in various types of structures. Recently, a new kind of materials called the FGP materials, have been developed to improve the reliability and effectiveness of piezoelectric structures by extending the concept of well-known Functionally Graded Materials (FGM) to piezoelectric materials. The emergence of FGP materials has demonstrated that they have the potential to reduce stress concentration and provide improved residual stress distribution, enhanced thermal properties, and higher fracture toughness. Beam-liked FGP structures are commonly used as sensors and actuators in a variety of mechanical, civil, and structural applications at various scales (Qin, 2013; Yang, 2005). Li et al. (2006) studied thermal post-buckling of Functionally Graded (FG) beams based on Timoshenko beam theory. They extracted nonlinear governing equations of the beam under non-uniform thermal and mechanical loads. Then, they evaluated thermal post-buckling of fixed-fixed beams by using a shooting method. Ying et al. (2008) studied bending and free vibration of an FG beam, which was located on the Winkler-Pasternak elastic substrate, using an analytical method. They investigated the effect of various parameters such as the power law index and aspect ratio on the response of the FG beam. Pradhan and Murmu (2009) explored vibration of the FG beam located on the Winkler elastic substrate by using a modified DQ (differential quadrature) method. Kiani and Eslami (2010) accomplished an analytical research into thermal buckling of FG beams, assuming that material properties changed according to the power law. They utilized the Euler-Bernoulli beam theory with consideration of nonlinear terms of strain in the formulation. In that paper, the critical temperature was obtained for three types of uniform, linear and nonlinear thermal loadings through the thickness direction of the beam. Doroushi et al. (2011) 
reported the dynamic response of FGPM beams based on the third-order shear deformation theory of a simple higher-order theory by using the finite element method. Fallah and Aghdam (2011) used the Euler-Bernoulli beam theory to study free vibration and post-buckling of the FG beams which were supported by a nonlinear elastic substrate. Furthermore, they assumed the von Kármán nonlinear strains in the formulation and solved the obtained governing equations of the beam by He's variational method. Wattanasakulpong et al. (2011) studied buckling and vibration of FG beams based on the third order shear deformation beam theory by using the power law model to define material properties through the thickness direction. They solved the eigenvalue problem by the Ritz method. Davoodinik and Rahimi (2011) investigated large deformation of tapered FG beams using a semi-analytical method. Li and Batra (2013) studied the buckling load of functionally graded Timoshenko and Euler-Bernoulli beams. They used the equilibrium method to derive governing equations of the FG beam and solved the obtained equations for different boundary conditions except a clamped-simply supported (C-S) beam. For C-S beams, they used a transcendental equation to find the critical buckling load. Zhang (2013) analyzed nonlinear bending of FGM beams based on the physical neutral surface and higher order shear deformation theory. He considered material properties to be temperature-dependent and variable in the thickness direction. Esfahani et al. (2013) studied non-linear thermal stability of temperature dependent FGM beams supported on non-linear hardening elastic foundations. They utilized a modified DQ method to solve the governing equations. They also explored some kinds of boundary conditions and thermal loading in analysis of the stability of FGM beams. Fu et al. (2012) investigated buckling, free vibration and dynamic stability of FGP beams in thermal environment by using nonlinear analysis. To perform thermal-electrical buckling solutions, they used the Euler-Bernoulli beam theory and Galerkin method. Komijani et al. (2013a) studied non-linear thermo-electrical stability of FGP beams based on the Timoshenko beam theory. They utilized the finite element method to analyze nonlinear behavior of beams in different boundary conditions. In an other work, Komijani et al. (2013b) investigated nonlinear stability and vibration of pre and post-buckled FGPM microstructures.

In this paper, thermal, mechanical and electrical loads are considered. A modified coupled stress theory and the von Kármán strains are utilized to obtain governing equations of the beam. Nasirzadeh et al. (2014) studied stability of FGP beams under thermal, electrical and mechanical loadings, and showed that thermal loading had a greater effect on the buckling point of the FGP beam in comparison with the electrical loading.

In this paper, stability and instability of FGP beams are investigated under thermal and electrical loadings. Material properties are considered to change gradually according to the power law. The governing equations are derived based on the Timoshenko beam theory. The FGP beam is under electrical, thermal and mechanical loadings. The temperature field is assumed to change uniformly and linearly in the thickness direction of the beam. The governing equation of the FGP beam is derived using the minimum potential theory and then the governing equation is solved by using an analytical method. Stability of the beam is investigated in the presence of thermal and electrical fields. The influence of effective parameters on the buckling load of the FGP beam such as: power law index, temperature field, applied voltage and aspect ratio is investigated.

\section{Theoretical formulation}

\subsection{Governing equations}

Figure 1 shows the proposed FGP beam of length $L$ and a rectangular cross section with thickness $h$ and width $b$ that is subjected to an axial compression load $P$. The coordinate axes are shown in Fig. 1. 

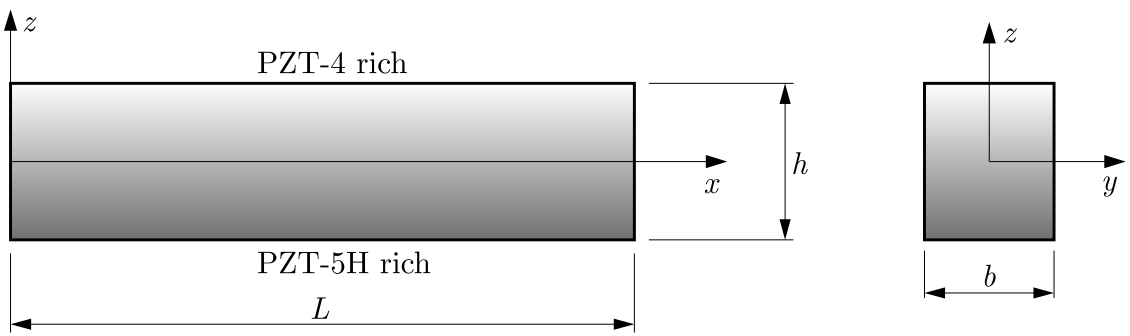

Fig. 1. FGP beam of length $L$ and rectangular cross section

The smooth and continuous distribution of material properties along thickness of the FGP beam, composed of two piezoelectric materials, follows a simple power law as (Komijani et al., 2013a)

$$
P(z)=P_{L}+P_{U L}\left(\frac{1}{2}+\frac{z}{h}\right)^{k}
$$

in which $P_{U L}=P_{U}-P_{L}$, where $P_{L}$ and $P_{U}$ are the material properties of lower and upper surfaces of the beam, respectively.

By applying a constant voltage to the FGP beam, an electric field is produced which can be defined as (Kiani and Eslami, 2010)

$$
E_{z}=-\frac{V_{0}}{h}
$$

In this paper, the governing equation is derived based on the Timoshenko beam theory. The displacements of an arbitrary point along the $z$ - and $x$-axes are denoted by $\widetilde{w}(x, z)$ and $\widetilde{u}(x, z)$, respectively. These displacements are formulated clearly as (Komijani et al., 2013b)

$$
\widetilde{u}(x, z)=u(x)-z \phi(x) \quad \widetilde{w}(x, z)=w(x)
$$

where $u(x)$ and $w(x)$ are displacements components in the mid-plane of the beam in the $z$ and $x$ direction, and $\phi$ is the rotation of plane of cross section.

From equations (2.3), the von Kármán type strains can be calculated as

$$
\varepsilon_{x}=u_{, x}+\frac{1}{2}\left(w_{, x}\right)^{2}-z \phi_{, x} \quad \gamma_{x z}=\phi+w_{, x}
$$

The constitutive equations of the FGP beam are derived considering the thermal and electrical fields as follows (Komijani et al., 2013a)

$$
\begin{array}{ll}
\sigma_{x}=Q_{11}(z)\left(\varepsilon_{x}-\alpha(z) \Delta \theta\right)-e_{31}(z) E_{z} & \tau_{x z}=Q_{55}(z) \gamma_{x z} \\
D_{z}=e_{31}(z) \varepsilon_{x}+k_{33}(z) E_{z}+p_{3} \Delta \theta & D_{x}=e_{15}(z) \gamma_{x z}
\end{array}
$$

in which $\sigma_{x}, \tau_{x z}, \varepsilon_{x}, \gamma_{x z}, D_{i}$ and $E_{z}$ are the axial stress, shear stress, axial strain, shear strain, electrical displacement and electrical field, respectively. Moreover, $Q_{i j}, \alpha_{i j}, e_{i j}, k_{i j}, p_{3}$ and $\Delta \theta$ are the elastic stiffness coefficient, thermal expansion coefficient, piezoelectric coefficient, dielectric coefficient, pyroelectric coefficient and temperature rise, respectively. In the current paper, the governing equations of the FGP beam subjected to mechanical, electrical and thermal loads are derived using the principle of minimum potential energy. Based on this principle, the equilibrium equations are derived when the following equation is satisfied (Komijani et al., 2013a)

$$
\delta \Pi=\delta H+\delta W_{e x t}=0
$$


in which $H$ is the electrical enthalpy and $W_{\text {ext }}$ is the virtual work of external forces imposed on the beam. The variation of electrical enthalpy for the FGP beam can be derived as (Kiani and Eslami, 2010)

$$
\delta H=\iiint_{V}\left[\sigma_{x} \delta \varepsilon_{x}+K_{s} \tau_{x z} \delta y_{x z}-D_{z} \delta E_{z}\right] d V
$$

where $K_{s}$ is the shear correction coefficient and is equal here to $5 / 6$ (Bathe, 1996). It should be noted that since the electrical field is not varied, $E_{z}$ is constant, so $\delta E_{z}$ is equals to zero. The virtual work done by external forces can be calculated as (Ballas, 2007)

$$
\delta W_{e x t}=-\int_{0}^{L} q \delta w d x-\bar{P} \delta u-\bar{M} \delta w_{, x}-\bar{R} \delta w
$$

The parameters, $\bar{R}, \bar{P}, \bar{M}$ are the axial resultant reaction force, supports external resultant reactions and external moment resultant reactions applied at the ends of the beam, respectively. Also, $q$ is the transversally distributed applied load. Based on Timoshenko beam theory, the stress resultant forces of the beam are derived using equations (2.7) and (2.8) as

$$
\begin{aligned}
& N_{x}=A_{11} u_{, x}-B_{11} w_{, x x}-N_{x}^{T}-N_{x}^{e} \\
& M_{x}=B_{11} u_{, x}-D_{11} w_{, x x}-M_{x}^{T}-M_{x}^{e} \\
& Q_{x}=K_{s} A_{55}\left(\phi+w_{, x}\right)
\end{aligned}
$$

where $N_{x}^{T}$ and $M_{x}^{T}$ are the corresponding thermal force and moment. Furthermore, $D_{11}, B_{11}$, $A_{11}$ are tension stiffness, tension bending and bending coefficients, which are defined as

$$
\left(A_{11}, B_{11}, D_{11}\right)=\int_{-h / 2}^{h / 2} Q_{11}(z)\left(1, z, z^{2}\right) d z \quad A_{55}=\int_{-h / 2}^{h / 2} Q_{55}(z) d z
$$

Also, the thermal force and resultant moment can be calculated as

$$
\left(N_{x}^{T}, M_{x}^{T}\right)=\int_{-h / 2}^{h / 2} Q_{11}(z) \alpha(z) \Delta \theta(1, z) d z
$$

Finally, the electrical force resultants can be written as

$$
\left(N_{x}^{e}, M_{x}^{e}\right)=\int_{-h / 2}^{h / 2} e_{31}(z) E_{z}(1, z) d z
$$

Substituting equations (2.7) and (2.8) into (2.6) and integrating with respect to $z$, and substituting of equation (2.9) and (2.10), the equilibrium equations for the beam have been derived as

$$
N_{x, x}=0 \quad Q_{x, x}+N_{x} w_{, x x}=0 \quad M_{x, x}-Q_{x}=0
$$

Substituting equation (2.9) into equation (2.13) and doing some simplifications, an ordinary differential equation with respect to displacement will be obtained. The final governing equation of the FGP beam based on Timoshenko assumptions is

$$
w_{, x x x x}+\mu^{2} w_{, x x}=0
$$


where

$$
\mu^{2}=\frac{A_{11} N_{x}}{\left(B_{11}^{2}-A_{11} D_{11}\right)\left(1+\frac{N_{x}}{K_{s} A_{55}}\right)}
$$

Equation (2.14) is a forth order ordinary differential equation which describes deflection of the beam.

\subsection{Boundary conditions}

The corresponding boundary conditions are considered as

$$
\begin{array}{ll}
N_{x}=\bar{P} & \text { or } \quad u=0 \\
w_{, x x x}+\mu^{2} w_{, x}=\frac{\bar{R} \mu^{2}}{N_{x}} & \text { or } \quad w=0 \\
M_{x}=\frac{1}{A_{11}}\left[\left(A_{11} D_{11}-B_{11}^{2}\right) \phi_{, x}+B_{11} P\right]-M_{x}^{T}-M_{x}^{e}=0 & \text { or } \quad \phi=0
\end{array}
$$

Using equations (2.9) and (2.16) yields

$$
\bar{P}=P-N_{x}^{T}-N_{x}^{e} \quad \bar{M}=M-M_{x}^{T}-M_{x}^{e} \quad \bar{R}=R-R_{x}^{T}-R_{x}^{e}
$$

in which the parameters $P, R, M$ are the external axial force, reaction force from supports and external moment applied at the ends of the beam, respectively. Also, the parameters $\bar{P}, \bar{R}, \bar{M}$ are the axial resultant reaction force, resultant reactions from external supports and external resultant moment at the ends of the beam, respectively. The other parameters are the thermal and electrical resultant forces defined in Eqs. (2.11) and (2.12). The formulas for each type of boundary conditions are listed in Table 1.

Table 1. The boundary conditions for the FGP beam

\begin{tabular}{|l|c||}
\hline Boundary conditions & $x=0$ or $l$ \\
\hline \hline Clamped & $w=\phi=0$ \\
\hline Simply supported & $w=\left(A_{11} D_{11}-B_{11}^{2}\right) \phi_{, x}+B_{11} P-A_{11}\left(M_{x}^{T}+M_{x}^{e}\right)=0$ \\
\hline Roller & $w_{, x x x}+\mu^{2} w_{, x}=\phi=0$ \\
\hline
\end{tabular}

\section{Solution}

The exact solution to differential equation (2.14) based on the parameter $\mu$, which depends on thickness and the resultant axial force of the beam, can be written as

$$
w(x)=C_{1} \sin (\mu x)+C_{2} \cos (\mu x)+C_{3} x+C_{4}
$$

where constants $C_{1}-C_{4}$ are calculated by using the boundary condition of the FGP beam. In order to deal with the constants, based on the Timoshenko beam theory and the number of the coefficient, we need to evaluate the deflection and slope of the beam in each boundary. Thus, the slope function can be presented using the deflection of the beam as

$$
\phi(x)=\left(1+\frac{N_{x}}{K_{s} A_{55}}\right)\left[-C_{1} \mu \cos (\mu x)+C_{2} \mu \sin (\mu x)\right]-C_{3}
$$

In this paper, stability of five types of boundary conditions is studied. The results show that instability occurs in two cases of boundary conditions (clamped-clamped (C-C) and clamped- 
-roller (C-R)) while three others (simply supported-simply supported (S-S), simply supported-clamped (S-C), simply supported-roller (S-R)) are stable. For an example, the C-C condition is considered as a sample of instability in the beam. These results are the same for two different Euler-Bernoulli and Timoshenko beam theories and the obtained results can be validated by the data reported by Nasirzadeh et al. (2014). In Table 2, the effect of the boundary condition on stability of the FGP beam is abstracted.

Table 2. The effect of the boundary condition on stability of the FGP beam

\begin{tabular}{|l|c|c|c|c|c|}
\hline Boundary conditions & C-C & C-R & S-S & S-C & S-R \\
\hline Stability behavior & unstable & unstable & stable & stable & stable \\
\hline
\end{tabular}

To satisfy the boundary condition, the algebraic equation constants $C_{i}$ are obtained using equation (2.18)

$$
\left[\begin{array}{cccc}
0 & 1 & 0 & 1 \\
-\mu S & 0 & -1 & 0 \\
\sin (\mu L) & \cos (\mu L) & L & 1 \\
-\mu S \cos (\mu L) & \mu S \sin (\mu L) & -1 & 0
\end{array}\right]\left[\begin{array}{l}
C_{1} \\
C_{2} \\
C_{3} \\
C_{4}
\end{array}\right]=\left[\begin{array}{l}
0 \\
0 \\
0 \\
0
\end{array}\right]
$$

where

$$
S=1+\frac{N_{x}}{K_{s} A_{55}}
$$

System of equations (2.20) has infinitely many non-trivial solutions if its coefficient matrix is singular. The non-trivial solution is obtained by equaling the determinant of the coefficient matrix to zero. By solving the obtained characteristic equation, it can be possible to determine the buckling load of the FGP beam for the C-C boundary condition. The characteristic equation of the coefficient matrix can be written as

$$
S \mu(L S \mu \sin (\mu L)+2 \cos (\mu L)-2)=0
$$

By solving equation (2.22), the buckling load of the FGP beam can be obtained as

$$
(\bar{P})_{c r}=\left(P-N_{x}^{T}-N_{x}^{e}\right)_{c r}=\frac{\frac{4 n^{2} \pi^{2}}{L^{2}}\left(B_{11}^{2}-A_{11} D_{11}\right)}{A_{11}-\frac{4 n^{2} \pi^{2}}{L^{2}} \frac{B_{11}^{2}-A_{11} D_{11}}{K_{s} A_{55}}} \quad n=1,2, \ldots
$$

Based on equation (2.23), the buckling load of the beam subjected to mechanical, thermal and electrical loads will be obtained. For the second example, the case of S-S boundary condition is considered as a sample of stable behavior of the structure. Like in the previous example, by applying the boundary conditions, the algebraic equations of the beam will be derived as

$$
\left[\begin{array}{cccc}
0 & 1 & 0 & 1 \\
0 & S \mu^{2} & 0 & 0 \\
\sin (\mu L) & \cos (\mu L) & L & 1 \\
S \mu^{2} \sin (\mu L) & S \mu^{2} \cos (\mu L) & 0 & 0
\end{array}\right]\left[\begin{array}{l}
C_{1} \\
C_{2} \\
C_{3} \\
C_{4}
\end{array}\right]=\left[\begin{array}{l}
0 \\
1 \\
0 \\
1
\end{array}\right] \frac{A_{11}\left(M_{x}^{T}+M_{x}^{e}\right)-B_{11} P}{A_{11} D_{11}-B_{11}^{2}}
$$

The above equation has not any non-trivial solution and hence the structure shows stable behavior. By solving equation (2.24), the deflection of the beam is obtained as

$$
w(x)=\frac{F}{S \mu^{2}}\left(\frac{1-\cos (\mu L)}{\sin (\mu L)} \sin (\mu x)+\cos (\mu x)-1\right)
$$


in which

$$
F=\frac{A_{11}\left(M_{x}^{T}+M_{x}^{e}\right)-B_{11} P}{A_{11} D_{11}-B_{11}^{2}}
$$

It should be noted that the nonhomogeneous distribution of the material through thickness of the beam can lead to inhomogeneous boundary conditions, and so, the beam shows stable behavior.

\subsection{Temperature field}

In this paper, two kinds of thermal fields acting on the beam are investigated. In the first case, the beam is subjected to a uniform temperature field with the temperature rise of $\Delta \theta=\theta-\theta_{0}$, in which $\theta_{0}$ is the initial temperature and $\theta$ is the final temperature imposed on the beam. In the second case, the beam is subjected to a linear temperature field through thickness of the beam. Using the assumption of a thin beam and solving the obtained heat transfer equation, the temperature distribution in the beam is derived as (Bodaghi et al., 2014)

$$
\theta=\theta_{L}+\theta_{U L}\left(\frac{1}{2}+\frac{z}{h}\right) \quad \theta_{U L}=\theta_{U}-\theta_{L} \quad \Delta \theta=\theta-\theta_{0}
$$

where $\theta_{L}$ and $\theta_{U}$ are temperatures of the lower and upper surfaces of the FGP beam, respectively.

\section{Results and discussions}

Consider a beam composed of a functionally graded material of PZT-4 and PZT-5 in the upper and lower surfaces, respectively. The properties of materials are listed in Table 3. In this Section, the buckling load of the FGP beam subjected to mechanical, thermal and electrical fields is studied. In the following Section, the results for Euler-Bernoulli and Timoshenko beams have been calculated and compared to each other. Moreover, the effect of uniform and linear thermal fields on the buckling load of the Timoshenko beam has been investigated. Finally, the critical values of the buckling load for the FGP beam with the clamped-clamped boundary conditions in thermal and electrical environment for different power law indexes and various aspect ratios will be studied.

Table 3. Thermal-electrical and mechanical properties of PZT-4 and PZT-5H (Komijani et al. 2013a)

\begin{tabular}{|l|c|c|}
\hline \multicolumn{1}{|c|}{ Property } & PZT-5H & PZT-4 \\
\hline \hline$Q_{11}[\mathrm{GPa}]$ & 60.6 & 81.3 \\
\hline$Q_{55}[\mathrm{GPa}]$ & 23.0 & 25.6 \\
\hline$e_{13}\left[\mathrm{C} / \mathrm{m}^{2}\right]$ & -16.604 & -10.0 \\
\hline$e_{15}\left[\mathrm{C} / \mathrm{m}^{2}\right]$ & 44.9046 & 40.3248 \\
\hline$k_{11}\left[\left(\mathrm{C}^{2} / \mathrm{m}^{2} \mathrm{~N}\right) \cdot 10^{-8}\right]$ & 1.5027 & 0.6712 \\
\hline$k_{33}\left[\left(\mathrm{C}^{2} / \mathrm{m}^{2} \mathrm{~N}\right) \cdot 10^{-8}\right]$ & 2.554 & 1.0275 \\
\hline$\alpha[1 / \mathrm{K}]$ & $10 \mathrm{E}-6$ & $2 \mathrm{E}-6$ \\
\hline$p_{3} \cdot 10^{-5}$ & 0.548 & 2.5 \\
\hline
\end{tabular}

At first, we compare our exact results with other data reported in the literature. Table 4 shows the buckling load of the piezoelectric beam for two different aspect ratios. The second solution is based on the finite element method. We used the two node Hermit elements and Euler-Bernoulli beam theory to model the beam. The results obtained by the FE method are 
Table 4. Comparison of the buckling load $(\mathrm{N} / \mathrm{m})$ of the clamped-clamped piezoelectric beam versus aspect ratio

\begin{tabular}{|c|c|c|}
\hline$L / h$ & Exact value (our paper) & FEM results \\
\hline \hline 25 & $3.1899 \mathrm{E}+06$ & $3.1899 \mathrm{E}+06$ \\
\hline 50 & $7.9746 \mathrm{E}+05$ & $7.9747 \mathrm{E}+05$ \\
\hline
\end{tabular}

compared with the exact solution which is obtained in this paper. As it is seen, the results for two distinct methods are in good agreement with each other.

Figure 2a shows the buckling load of the FGP beam versus the power low index $k$ for different aspect ratios $(L / h)$ for Euler-Bernoulli and Timoshenko beams. It is seen that by increasing the power law index $k$, the value of the buckling load increases. Also this figure shows that the load decreases with the increasing aspect ratio $L / h$. On the other hand, the differences between the buckling load obtained by using Euler-Bernoulli and Timoshenko beam theories are decreased when the ratio of $L / h$ is increased. It is seen that the value of the resultant axial buckling load predicted by the Euler-Bernoulli theory is higher than that by the Timoshenko beam theory. The reason can be explained by considering the effect of shear stresses in the Timoshenko beam theory. In Fig. 2b, variation of the buckling load versus aspect ratio of the beam for various power low indexes is depicted. As it is seen, by decreasing the aspect ratio, the buckling load increases.
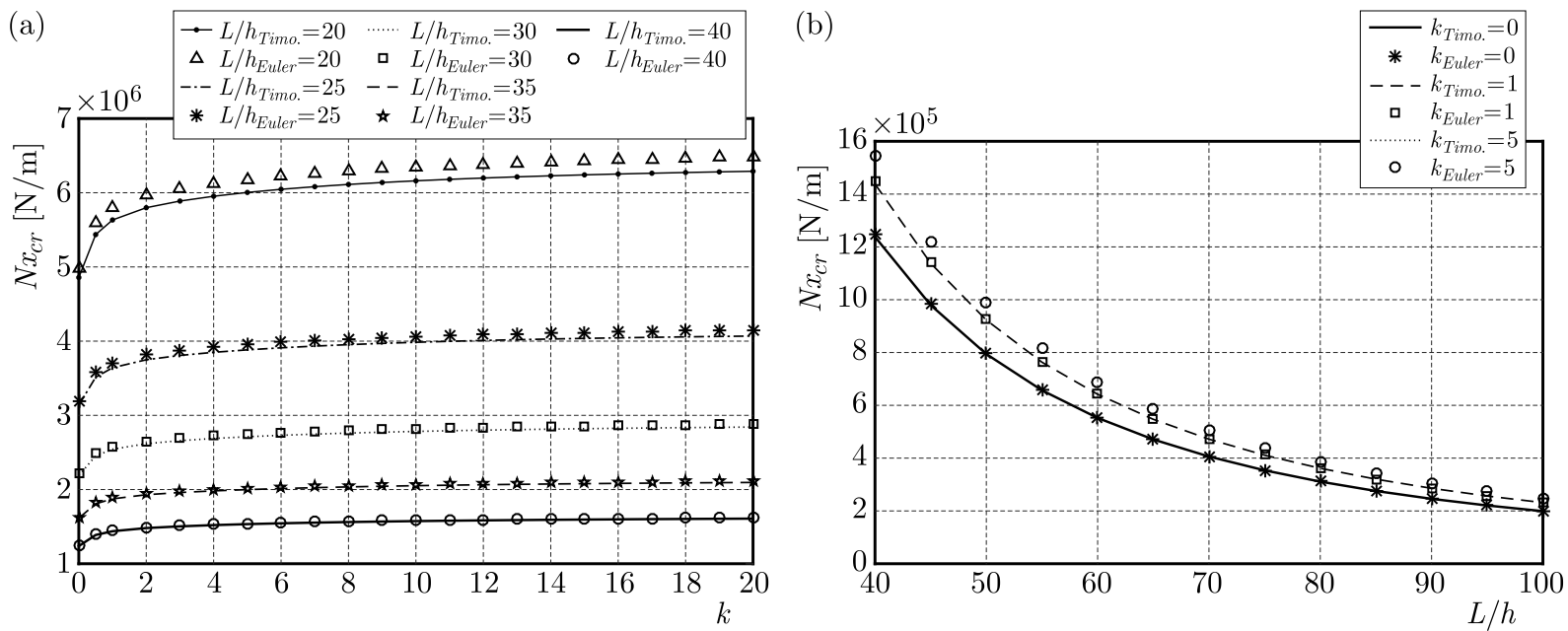

Fig. 2. (a) The effect of the power law index on the buckling load for various aspect ratios for Euler-Bernoulli and Timoshenko theories. (b) The effect of aspect ratios on the critical buckling load versus the power load index for Euler-Bernoulli and Timoshenko beam theories

In Figs. 3a and 3b, the effects of uniform and linear thermal fields on the buckling load of the beam are depicted. In the studied cases, the FGP beam exposed to a constant applied voltage $\left(V_{0}=500 \mathrm{~V}\right)$ and the aspect ratio of the beam is $(L / h=50)$. The results show that the rate of change of the buckling load for power law indexes between zero to four is high, and for power indexes which are more than four is decreased and changes no more. Also it is seen that for a constant power law index, by increasing temperature in the beam, the buckling force decreases. The produced elongation caused by the temperature changes can be regarded as a reason for this phenomenon. It should be noted that, because of the acceptable conformity between the results of two theories, the mentioned points are same for the two beam theories.

Figures $4 \mathrm{a}$ and $4 \mathrm{~b}$ show changes of the buckling load with respect to uniform temperature rise through thickness for different power law indexes of two theories. Because of the decreasing equivalent thermal expansion coefficient of the FGP beam by increasing of the power law index, 

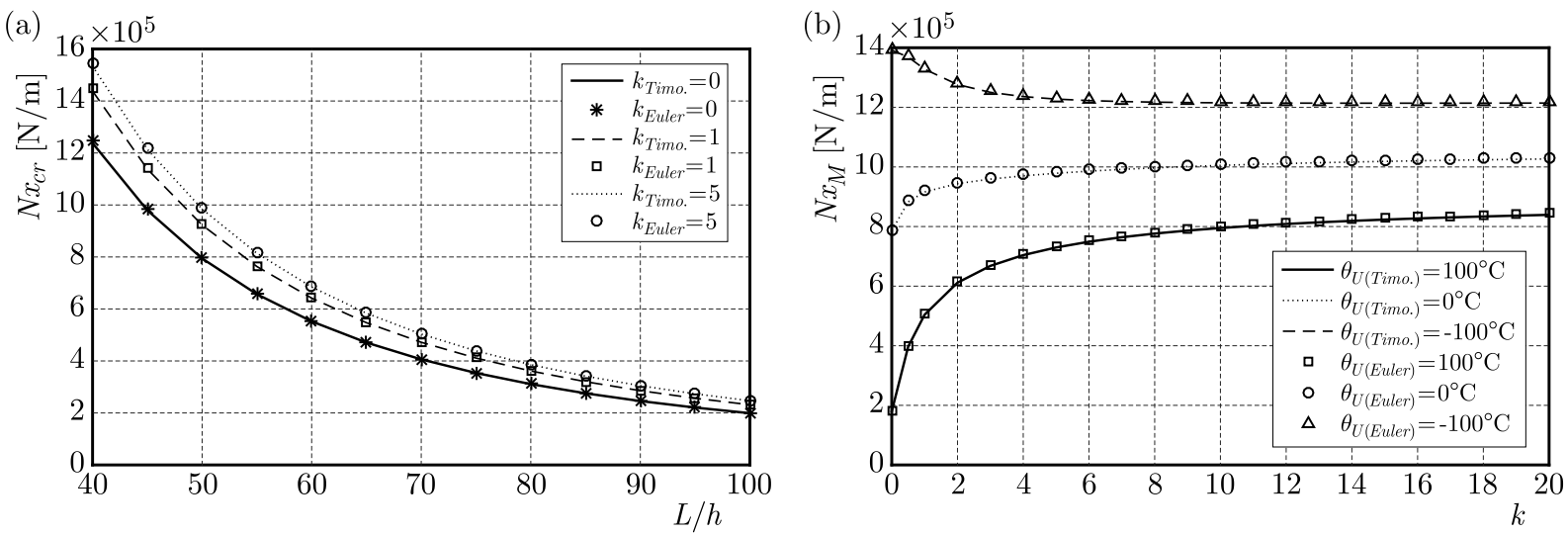

Fig. 3. (a) The effect of a constant thermal field on the buckling load with respect to the power index $k$ for two theories. (b) The effect of a linear thermal field along thickness on the buckling load versus the power index $k$ for two theories
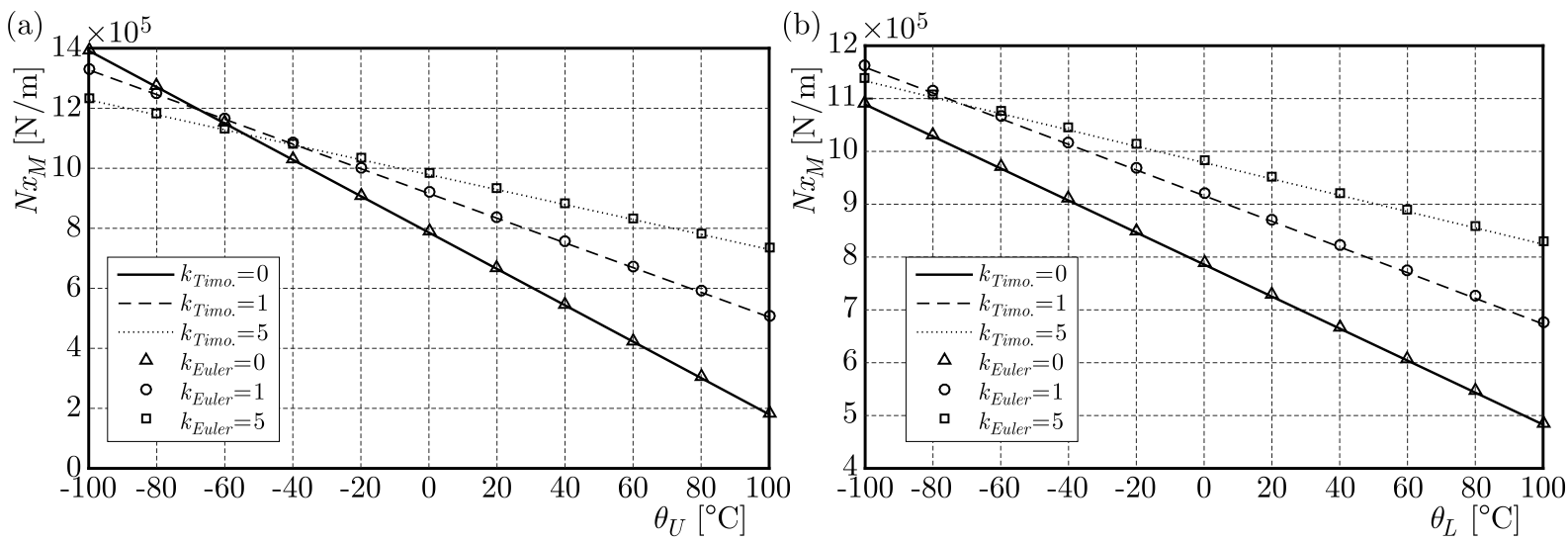

Fig. 4. (a) The effect of a constant thermal field on the buckling load for two theories for various power law indexes. (b) The effect of a linear thermal field on the buckling load for two theories for various power law indexes $\left(V_{0}=500 \mathrm{v}, L / h=50\right)$

the buckling load of the beam is decreased. Moreover, it should be noted that the intersection point of lines with various power indexes depends on the aspect ratio and thermal expansion coefficient of composed materials, and by changing of these parameters, this point is moved or eliminated.

In Fig. 5a, a comparison of the effects of the uniform and linear temperature rise through thickness of the Timoshenko beam on the buckling load is depicted. It can be seen that by increasing the temperature the critical buckling load is decreased. This phenomenon can be explained due to thermal stresses in the beam. The effect of the power law index on the critical buckling load for the uniform and linear temperature rise along the thickness of the beam for two theories are shown in Fig. 5b.

Figures 6, 7 and 8 show the effect of the power law index and applied voltage for the uniform and linear temperature rise on the critical buckling load of the beam. It can be inferred that in both conditions, by increasing the power law index, the critical buckling load is increased. Moreover, for a specified power law index, by decreasing the applied voltage, the value of critical buckling load increases; however it should be noted that the effect of voltage is negligible on the critical buckling load. Figure 6 shows the effect of uniform and linear temperature fields for different voltages on the critical buckling load. The most significant point is that the rate of buckling load variation by changing the applied voltage is almost negligible. It can be explained by the small value of the piezoelectric coefficient of the FGP beam. 

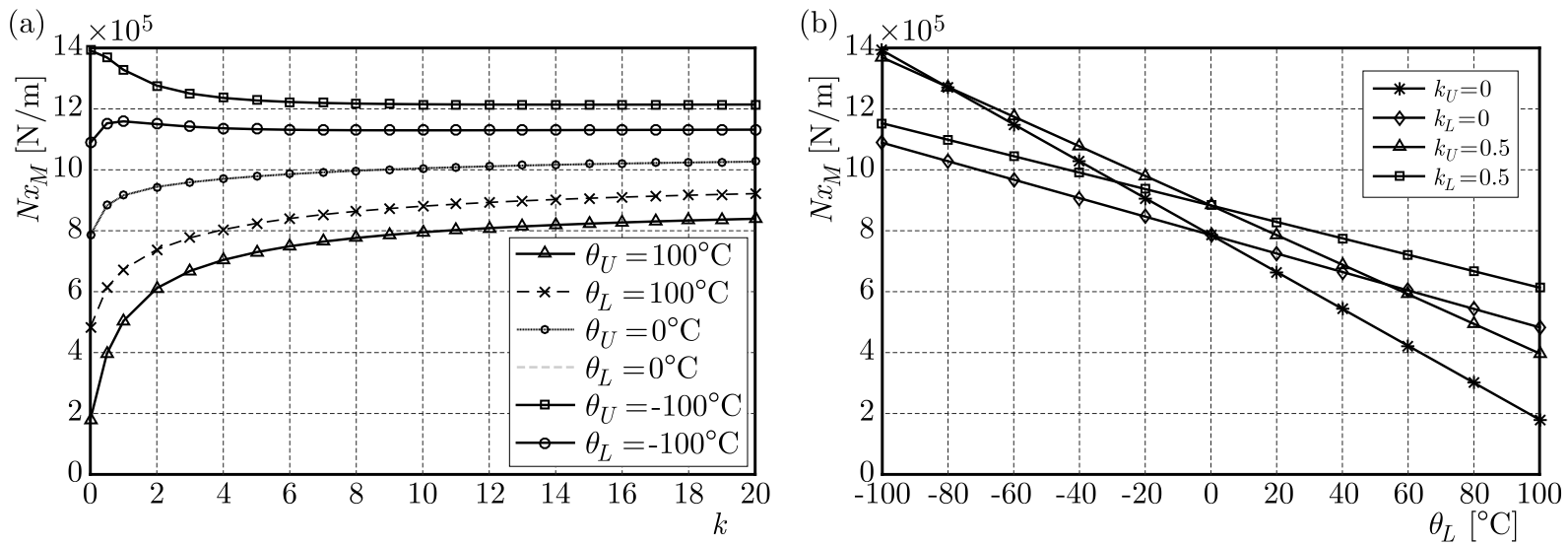

Fig. 5. (a) The effect of uniform and linear temperature fields on the buckling loads versus the power index for the Timoshenko beam. (b) The effect of uniform and linear thermal fields on the buckling load for various power indexes $\left(V_{0}=500 \mathrm{~V}, L / h=50\right)$
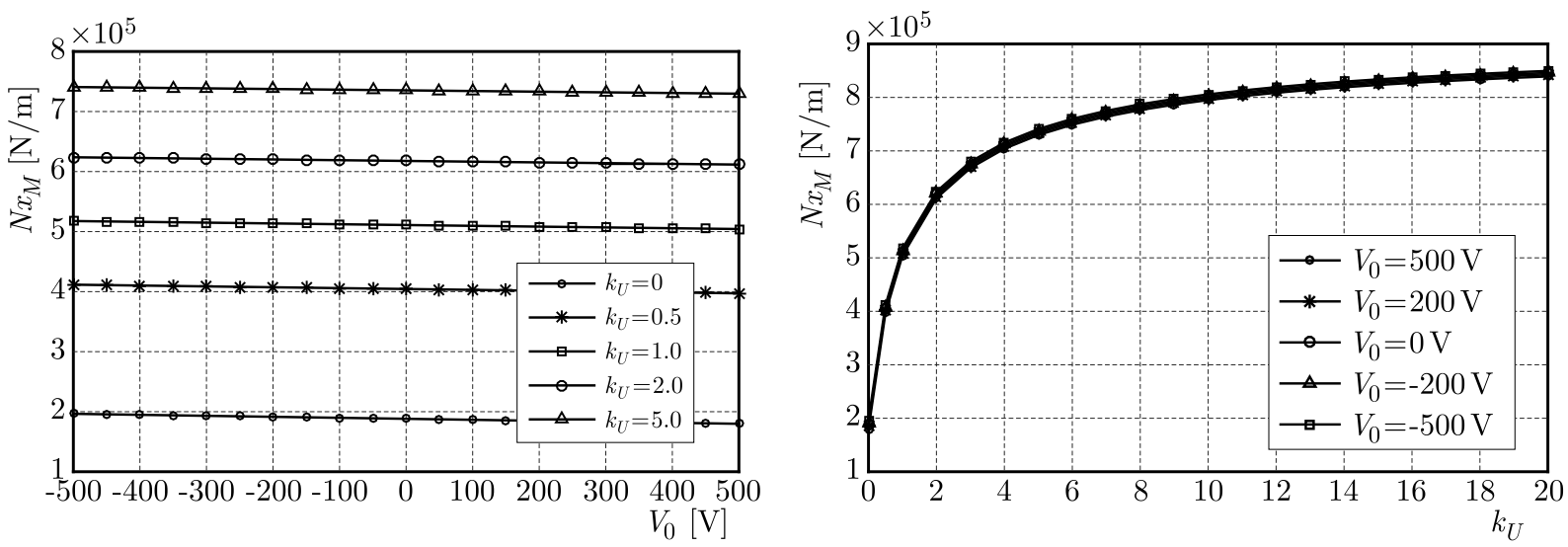

Fig. 6. The effect of voltage and power index on the critical buckling load for a uniform temperature rise
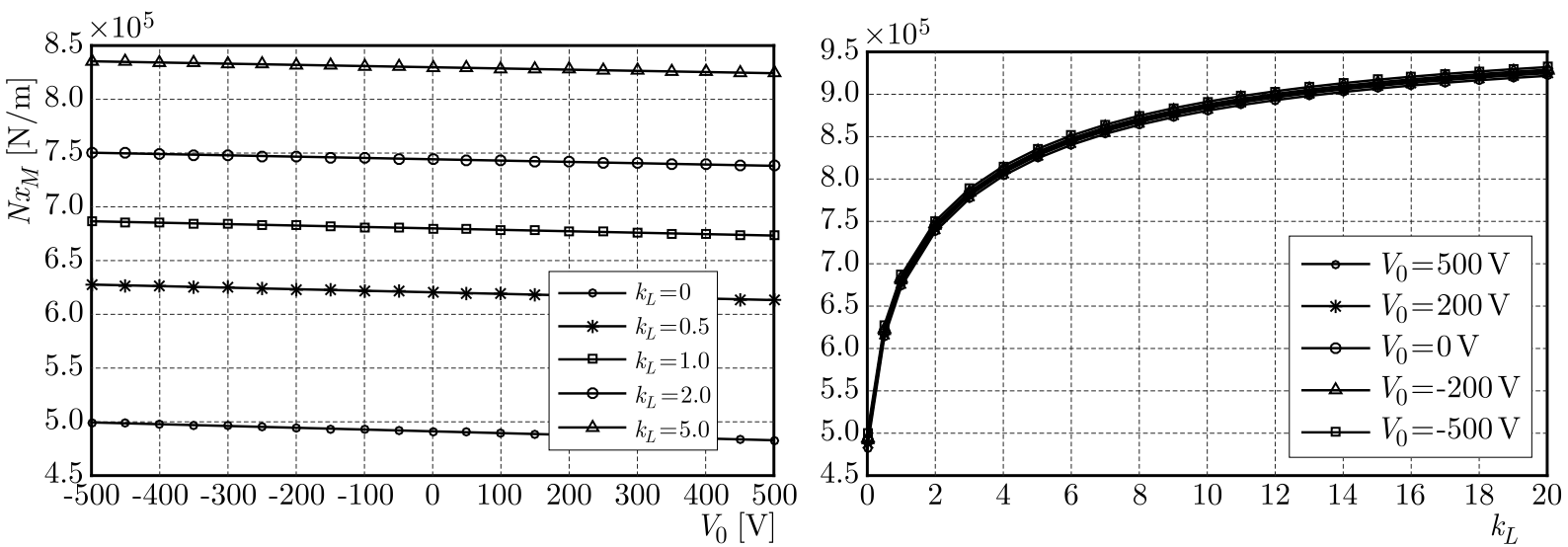

Fig. 7. The effect of voltage and power index on the critical buckling load for a linear temperature rise

Figure 9 shows the deflection of the FGPM beam with the S-S boundary condition versus the applied axial force. The beam is under a uniform thermal $(\Delta \theta=100)$ and electrical loading $\left(V_{0}=200 \mathrm{~V}\right)$. This figure shows that by increasing the applied force, the deflection do not change, but in points closer to the bifurcation point of the beam the deflection sharply increases. 

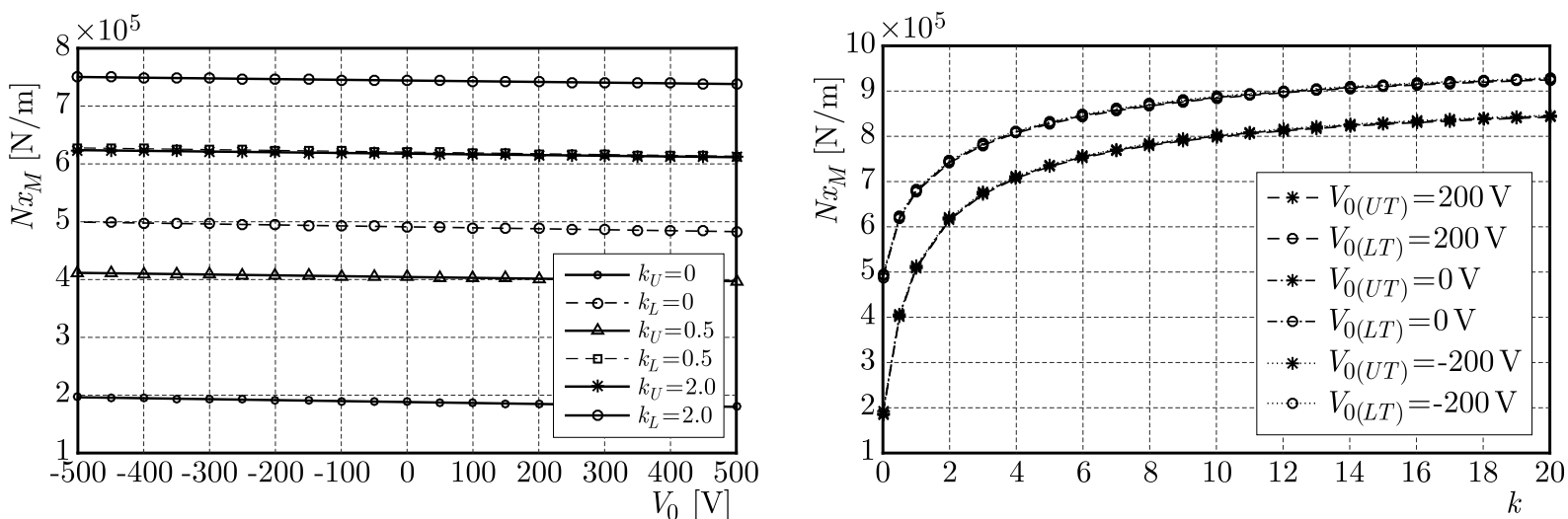

Fig. 8. The comparison of the critical buckling load for uniform and linear temperature field versus the applied voltage

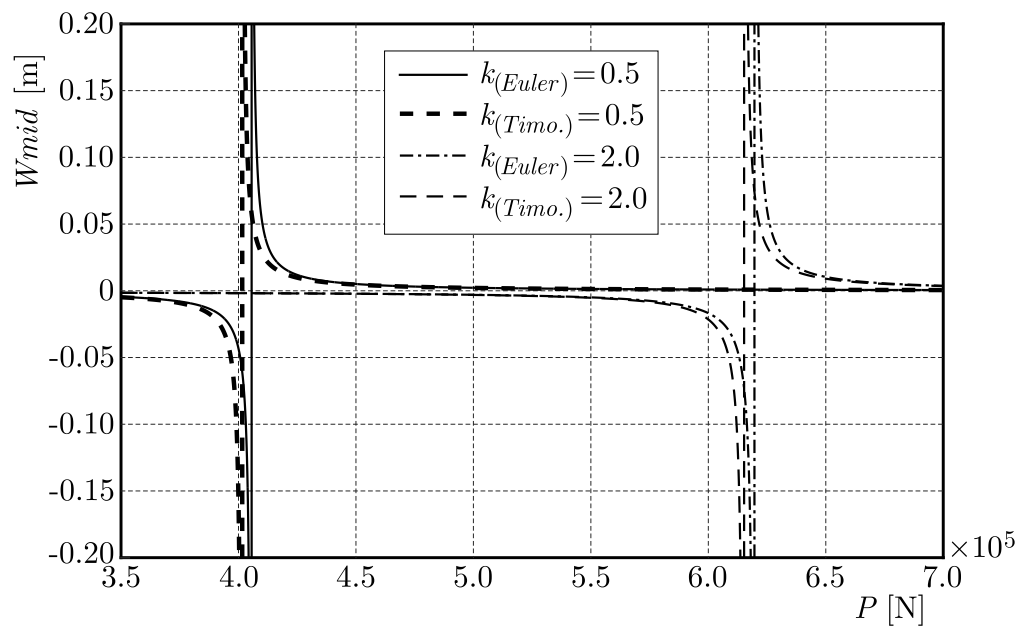

Fig. 9. Deflection of the SS beam under thermal $\left(\Delta \theta=100^{\circ} \mathrm{C}\right)$ and voltage load $\left(V_{0}=200 \mathrm{~V}\right)$

\section{Conclusions}

In this paper, the stability and instability of FGP beams is investigated based on the Timoshenko beam theory. Considering different boundary conditions of the FGP beam the instability is shown for two cases of boundary conditions: clamped-clamped (C-C) and clamped-roller (C-R). The three others: simply supported-simply supported (S-S), simply supported-clamped (S-C), simply supported-roller (S-R) show stable behavior. For both boundary conditions which are unstable (C-C and C-R), the results show that the buckling load increases with ithe ncreasing power law index. Also, by increasing the temperature, the value of the buckling load decreases. In addition, a uniform temperature rise has greater effect on the buckling load than a linear temperature rise. Moreover, the temperature field is more effective than the electric field in the buckling load of the beam, and the electrical loading has not a significant effect on the buckling load of the FGP beam.

\section{References}

1. Ballas R.G., 2007, Piezoelectric Multilayer Beam Bending Actuators: Static and Dynamic Behavior and Aspects of Sensor Integration, Springer

2. Bathe K.J., 1996, Finite Element Procedures, Prentice Hall 
3. Bodaghi M., Damanpack A.R., Aghdam M.M., Shakeri M., 2014, Geometrically non-linear transient thermo-elastic response of FG beams integrated with a pair of FG piezoelectric sensors, Composite Structures, 107, 48-59

4. Davoodinik A.R., Rahimi G.H., 2011, Large deflection of flexible tapered functionally graded beam, Acta Mechanica Sinica, 27, 5, 767-777

5. Doroushi A., Eslami M.R., Komeili, A., 2011. Vibration analysis and transient response of an FGPM beam under thermo-electro-mechanical loads using higher-order shear deformation theory, Journal of Intelligent Material Systems and Structures, 22, 3, 231-243

6. Esfahani S.E., Kiani Y., Eslami M.R., 2013, Non-linear thermal stability analysis of temperature dependent FGM beams supported on non-linear hardening elastic foundations, International Journal of Mechanical Sciences, 69, 10-20

7. Fallah A., Aghdam M.M., 2011, Nonlinear free vibration and post-buckling analysis of functionally graded beams on nonlinear elastic foundation, European Journal of Mechanics-A/Solids, 30, $4,571-583$

8. Fu Y., WAng J., MaO Y., 2012, Nonlinear analysis of buckling, free vibration and dynamic stability for the piezoelectric functionally graded beams in thermal environment, Applied Mathematical Modelling, 36, 9, 4324-4340

9. Kiani Y., Eslami M.R., 2010, Thermal buckling analysis of functionally graded material beams, International Journal of Mechanics and Materials in Design, 6, 3, 229-238

10. Komijani M., Kiani Y., Eslami M.R., 2013a, Non-linear thermoelectrical stability analysis of functionally graded piezoelectric material beams, Journal of Intelligent Material Systems and Structures, 24, 4, 399-410

11. Komijani M., Reddy J.N., Ferreira A.J.M., 2013b, Nonlinear stability and vibration of pre/post-buckled microstructure-dependent FGPM actuators, Meccanica, 49, 2729-2745

12. Li S.R., BAtra R.C., 2013, Relations between buckling loads of functionally graded Timoshenko and homogeneous Euler-Bernoulli beams, Composite Structures, 95, 5-9

13. Li S.R., Zhang J.H., Zhao Y.G., 2006, Thermal post-buckling of functionally graded material Timoshenko beams, Applied Mathematics and Mechanics, 27, 803-810

14. Nasirzadeh R., Behjat B., Kharazi M., 2014, Stability of FGP beams under thermo-electro-mechanical loading, International Journal of Material Science Innovations, 2, 6, 164-177

15. Pradhan S.C., Murmu T., 2009, Thermo-mechanical vibration of FGM sandwich beam under variable elastic foundations using differential quadrature method, Journal of Sound and Vibration, 321, 1, 342-362

16. QIN Q.H., 2013, Advanced Mechanics of Piezoelectricity, Springer

17. Wattanasakulpong N., Gangadhara Prusty B., Kelly D.W., 2011, Thermal buckling and elastic vibration of third-order shear deformable functionally graded beams, International Journal of Mechanical Sciences, 53, 9, 734-743

18. YAng J.S., 2005, Introduction to the Theory of Piezoelectricity, Springer, New York

19. Ying J., LÜ C.F., Chen W.Q., 2008, Two-dimensional elasticity solutions for functionally graded beams resting on elastic foundations, Composite Structures, 84, 3, 209-219.

20. Zhang D.G., 2013, Nonlinear bending analysis of FGM beams based on physical neutral surface and high order shear deformation theory, Composite Structures, 100, 121-126 